\title{
UPGRADES AND AUTOMATION AT THE VATICAN ADVANCED TECHNOLOGY TELESCOPE (VATT)
}

\author{
S. R. Swindell ${ }^{1}$, C. S. Johnson ${ }^{2}$, M. H. Franz ${ }^{3}$, P. Kubanek ${ }^{4}$, K. W. Duffek ${ }^{5}$, and P. Gabor ${ }^{6}$
}

\begin{abstract}
The Vatican Advanced Technology Telescope (VATT) is a $1.8 \mathrm{~m}$ Gregorian telescope with an $\mathrm{f} / 1$ primary and an f/9 full system optics. It has been in operation since 1993 and some of its systems are showing their age. Here, we list the completed and planned upgrades for some of the aging systems. We focus on a new secondary controller and high resolution absolute encoders. Our objective is to automate telescope operation. We outline the expanded science potential, especially for surveys and Time Domain Astronomy. Finally, we examine new software that will assist in automation and describe some of the challenges involved in automating VATT.

\section{RESUMEN}

El Telescopio de Tecnología Avanzada del Vaticano (VATT) es un telescopio gregoriano de 1,8 m de diámetro con un primario de f/1 y una óptica de sistema total de f/9. Ha estado en funcionamiento desde 1993 y algunos de sus sistemas ya dan muestras de envejecimiento. Enumeramos las actualizaciones completadas y planificadas para algunos de los sistemas obsoletos. Nos centramos en una nueva controladora secundaria, así como en codificadores absolutos de alta resolución. Nuestro objetivo es automatizar el funcionamiento del telescopio. Destacamos el gran potencial científico, especialmente para cartografiados y estudios de fotometría rápida. Finalmente, examinamos nuevo software que ayudará en la automatización y se describen algunos de los desafíos involucrados en la automatización de VATT.
\end{abstract}

Key Words: telescopes

\section{INTRODUCTION}

The Vatican Advanced Technology Telescope (West et al. 1997, www.vovatt.org) is an $\mathrm{f} / 9$ (full system) $1.8 \mathrm{~m}$ aplanatic Gregorian at the Mount Graham International Observatory. In operation since 1992, it has been the engine of a wide range of science (Hubbard et al. 1996; Tomaney \& Crotts 1996; Bertola et al. 1998; Corsini et al. 1999; Flaccomio et al. 1999; Grazian et al. 2000; Stanek et al. 2001; Vega Beltrán et al. 2001; Pignatelli et al. 2001; Rettig et al. 2001; Romanishin et al. 2001, 2010; Taylor et al. 2004, 2005; Uglesich et al. 2004; van Zee et al. 2004; Tegler et al. 2005; Verbiscer et al. 2006; Černis et al. 2012, 2016; Straižys et

\footnotetext{
${ }^{1}$ Steward Observatory, University of Arizona, 933 N Cherry Ave., Tucson AZ, 85721-0065, USA (scottswindell@email.arizona.edu).

${ }^{2}$ Steward Observatory, University of Arizona, $933 \mathrm{~N}$ Cherry Ave., Tucson AZ, 85721-0065, USA.

${ }^{3}$ Vatican Observatory Research Group, University of Arizona, 933 N Cherry Ave., Tucson AZ, 85721-0065, USA.

${ }^{4}$ Large Binocular Telescope Observatory, University of Arizona, 933 N Cherry Ave., Tucson AZ, 85721-0065, USA.

${ }^{5}$ MMT Observatory, University of Arizona, 933 N Cherry Ave., Tucson AZ, 85721-0065, USA.

${ }^{6}$ Vatican Observatory Research Group, University of Arizona, 933 N Cherry Ave., Tucson AZ, 85721-0065, USA (pgabor@as.arizona.edu).
}

al. 2013, 2014a,b,c, 2015; Harding et al. 2013). Its incremental upgrades, starting in 2010, are geared towards automated operation. Action points in progress include the installation of

- secondary mirror hexapod positioner,

- high-precision 26 bit encoders,

- intelligent servo drives,

- ladder logic safety system,

- new computer servers and network upgrade,

- software upgrades and RTS2 integration.

These measures will enable us to pursue new research programs with VATT as a stand-alone telescope and within a telescope network.

\section{UPGRADE PHILOSOPHY}

Since 2010, VATT has been capable of remote operation from campus with on-site support for opening and shutdown procedures, as well as for safety throughout the night. Since 2013, VATT has been gradually undergoing a general overhaul, necessary after 25 years in service. The goal is to allow for safe 
autonomous operation (automated queue and/or remote observing, including automated start-up and shut-down, without dedicated on-site support but with emergency support from third-party on-site staff). The guiding principle is distributed processing and modular design. Each subsystem will be controlled by a Local Control Unit (LCU) with a single-board computer or microcontroller (in practice, Arduino-based PLC), allowing a level of autonomy, making the system more robust as a whole.

This work is done in the context of a larger project, the Arizona Robotic Telescope Network (ARTN). It is a collaboration between University of Arizona's Steward Observatory (SO) and the Vatican Observatory (VO). The goal is to create a single facility initially comprising three telescopes: 61 " Kuiper Telescope on Mt Bigelow, 90" Bok Telescope on Kitt Peak, and 72" VATT on Mt Graham. The telescopes will be capable of autonomous operation without a human operator (with the exception of day-time inspections, supply of liquid nitrogen for the detectors, and instrument changes), running several science programs concurrently, including synoptic observations of the same object at the same time using three different instruments.

The mount control system for the telescope will be the TCSNG (Next Generation Telescope Control System), developed at SO. It will interface with a Facility Control System (FCS; controlling the infrastructure, as well as the safety and security of the operation) via secure network. Ladder logic circuits ensure safe operation on hardware level. One of the guiding principles of VATT automation is to implement essential features redundantly both in hardware and in software, first and foremost to ensure safety of persons and equipment.

This architecture will be mapped by RTS2 (www . rts2.org) drivers, enabling autonomous operation, scripting, scheduling (generating a target list based on observer and administrator determined priorities), coordination with other telescopes in the network.

\section{PLANNED AND COMPLETED UPGRADES}

\subsection{Secondary Mirror Hexapod}

Due to the fast optical system at VATT (f/1 primary mirror), an actively collimating secondary is necessary to maintain image quality. Piston (focus) and lateral displacement of the secondary mirror (M2) are sensitive to displacements of $<2 \mu \mathrm{m}$ and its orientation $\left(\operatorname{tip}_{x}, \operatorname{tip}_{y}\right)$ to angular deviations of $<2$ arcsec. The struts supporting M2 sag as a function of elevation and expand \& contract as a function of temperature. To compensate, corrections to M2's position need to be applied constantly by an "autocollimation" routine.

The original M2 positioning system was based on a pseudo-hexapod built in-house in c. 1991. Its principal component was a block of titanium with machined incisions producing 6 , non-orthogonal axes of flexure with stepper-motor driven screws as actuators. It was effective but it had its drawbacks. The design of the pseudo-hexapod limited the range of motion. The coupling of axes and the consecutive execution of the individual components of the commanded displacement, making precise position control difficult and smooth motion along the path of travel impossible. The system was unacceptably slow for robotic operation, typically executing a requested displacement request of $10 \mu \mathrm{m}$ in tens of seconds. The main issue, however, was that the obsolete controller had failing electronics components, which, since 2016, only functioned if kept at $>80^{\circ} \mathrm{F}\left(27^{\circ} \mathrm{C}\right)$.

We procured the H-824 hexapod from Physik Instrumente. The body is aluminum and M2's cylindrical baffle barrel is steel. We designed and manufactured a mechanical interface to compensate for the differential thermal expansion while maintaining parallelism and eliminating lateral motion (thus the only displacement caused by temperature changes is along the main axis of symmetry). We ported the autocollimation routine to a new software platform and designed a new graphical user interface based on Instrument Neutral Distributed Interface (INDI; www . indilib.org) \& html, an architecture which we are implementing for all upgraded systems.

\subsection{TCSNG Mount LCU, Encoders and Drives}

The TCSNG with new intelligent servo drives and new incremental encoders was commissioned on the Kuiper 61" telescope, achieving satisfactory operation parameters, with a tracking error $<0.5$ arcsec (due to mechanical limitations) and pointing accuracy of 10 arcsec.

A similar system TCSNG Mount LCU with ELMO drives and an additional Arduino unit (Controllino Mega) supervising ladder logic relays was built for the VATT. Renishaw encoders for the elevation and azimuth axes are about to be installed using appropriate mechanical interfaces. The commissioning (first using a breadboard then at VATT and on the sky) is scheduled for summer 2018, including flexure mapping, autocollimation, autofocusing, pointing, and guiding. Mount control safety systems (first and foremost the altitude \& azimuth breaks and stow pins controller) need to be developed. 


\subsection{IT Upgrades}

New servers (2 file servers HP ProLiant DL180 Gen9 and 4 servers HP ProLiant DL160 Gen9) and other IT equipment (ethernet switches, uninterruptible power supplies, etc.) were procured and installed.

The network was upgraded with new hardware (switches, uninterruptible power supplies - UPS, etc.) and its architecture was made more robust. We have a upgraded the router and we are enhancing VATT's redundant connection to the Internet with a link to data service via a commercial cellular telephone system. We have separated the user network from the telescope/instrument network, allowing the former to stay on during lightning shutdown. The telescope/instrument network has two main switches, each with its own UPS and each redundantly ensuring the connectivity of all network nodes (servers, TCSNG mount, etc.). The network within each node has a star topology.

\subsection{RTS2 Development for ARTN}

A version of RTS2 low-level and high-level software (device drivers and scheduler, respectively) was implemented on a number of Steward Observatory's telescopes (Raymond E. White, Jr. 21" Telescope on campus, Kuiper 61" Telescope and 27" Schmidt camera on Mt Bigelow, 40" and 60" on Mt Lemmon). Most of the RTS2 development work now takes place at the Kuiper 61" and focuses on queue management, high level graphical interfaces and overall safety of a robotic operation. The effort is made more challenging because the telescope is a 1960's design and it must remain operational for classical observing.

Currently, we run simple first-in, first-out queues while the observatory is monitored by an operator or engineer. In this mode, the majority of the work is done by RTS2. The operator attends to software crashes and bad weather. By the summer of 2018, we hope to run more complex queueing systems, e.g., intricate cadences and interruptions by targets of opportunity. This will be done primarily using RTS2's scripting capability (Kubánek et al. 2008). The ability to run the telescope without supervision is a challenge and will require more hardware automation and a series of safety audits.

\subsection{Future Action}

Planned action points include: The field derotator TCSNG routines; mirror cover, stow pins, counterweights, dome and slit control systems; new thermocouples with an appropriate electronics interface, monitoring the temperature of the mirror, mirror cell, and struts; in the guider system smart motors and LCU; hydrostatic oil bearing LCU; upgrades to the weather system with additional sensors; interlocks and other safety systems; and the integration and commissioning of the FCS.

\section{SCIENCE CASES}

VATT has a CCD imager (12 arcmin square, $4 \mathrm{k} \times 4 \mathrm{k}$ pixels) and a slit spectrograph (covering $360-950 \mathrm{~nm}$; three gratings giving resolutions of 6000,3000 and 1500 , i.e., $0.1,0.2$, and $0.4 \mathrm{~nm}$, resp.), both from Steward Observatory's Imaging Technology Laboratory, using AzCam software.

In addition, VATT can be used with the Potsdam Echelle Polarimetric and Spectroscopic Instrument (PEPSI; Strassmeier et al. 2015) located in the Large Binocular Telescope pier and connected via optical fiber. Since 2014, the VATT-PEPSI connection has been used for 40 nights a year, facilitating PEPSI commissioning. In 2018-2020, we shall devote 150 VATT nights to high-resolution spectroscopic survey $(\mathrm{R}=200,000)$ of $311 \mathrm{~V} 8.5 \mathrm{mag}$, cooler than F0 main-sequence stars in the $800^{\circ 2}$ around the North Ecliptic Pole. We shall determine their radial velocity (to $2-3 \mathrm{~m} / \mathrm{s}$ ), effective temperature, gravity, metallicity, chemical abundances, etc. in preparation for the TESS mission (Ricker et al. 2015). In this study VATT's systems will mainly profit from the upgrade of the secondary mirror's positioning system. With typical integration times of $90 \mathrm{~min}$, and guiding with a dedicated fiber injection unit (Strassmeier et al. 2015), the other upgrades will not play a critical role.

Starting in late 2018, VATT with its CCD imager is envisaged to take part in a survey of nearby $\mathrm{M}$ dwarfs in search of transiting exo-Earths. In this case, integration times will be less than $2 \mathrm{~min}$, and improvements in pointing (3.2) will greatly benefit the program.

In both cases, a shift in the role of human operators from manual control of the telescope to supervision of its automated operation, will lessen the strain on human resources and allow us to involve undergraduate students in the projects.

The developing Arizona Robotic Telescope Network will pursue a number of science cases:

- Supernovae: type Ia and IIn (photometry)

- Gamma-Ray Bursts (photometry)

- Reverberation Mapping (photometry)

- Asteroids: astrometric follow-up and photometric and spectroscopic characterization (astrometry, photometry and spectroscopy) 
- Quasar candidate spectroscopy

- Space situational awareness

VATT's role within ARTN remains to be specified. At least in the first stage, each ARTN telescope cannot pursue automated network operation with more than one instrument. Among ARTN assets, VATT is particularly well placed to focus on spectroscopic observations. If we commit to this program we shall have to make VATT's spectrograph more robust (its calibration sources in particular).

\section{CONCLUSION}

VATT's upgrades and automation are gradual and incremental. The important achievements of the past months have been M2's hexapod positioner and the upgrades to the IT infrastructure.

The major milestone for summer 2018 shutdown period is the transition from VATT's current encoders, drives and legacy TCS to the new encoders, drives and TCSNG. It is realistic to expect that this will reduce pointing and tracking errors to subarcsecond levels.

We are preparing to upgrade the guider system in the observing season of 2018/2019 building a new LCU and using already procured smart motors (with absolute encoders). We are also planning to implement a state machine which will greatly facilitate the startup and shutdown procedures, provide centralized system state logging as well as enhance the safety of VATT's operation. At first it will supervise VATT's mount and other LCU-run systems, including some aspects of the FCS. The systems temporarily relying on human intervention will also benefit from the state machine, guiding the operator and logging the action.

We hope to reach the goal of safe autonomous operation ( $(2)$ by 2020 , anticipating that thorough commissioning of the FCS will be the last stage of the process.

\section{REFERENCES}

Bertola, F., Cappellari, M., Funes, S. J., José G., et al. 1998, ApJ, 509, L93

Černis, K., Boyle, R. P., Laugalys, V., \& Wlodarczyk, I. 2012, BaltA, 21, 455

Černis, K., Boyle, R. P., \& Wlodarczyk, I. 2016, BaltA, 25,189
Corsini, E. M., Pizzella, A., Sarzi, M., et al. 1999, A\&A, 342,671

Flaccomio, E., Micela, G., Sciortino, S., et al. 1999, A\&A, 345,521

Harding, L. K., Hallinan, G., Boyle, R. P., et al. 2013, ApJ, 779, 101

Hubbard, W. B., Consolmagno, G. J., Boyle, R. P., \& Hill, R. 1996, LPSC, 27

Grazian, A., Cristiani, S., D'Odorico, V., Omizzolo, A., \& Pizzella, A. 2000, AJ, 119, 2540

Kubánek, P., Jelínek, M., French, J., et al. 2008, SPIE, 7019, 2

Pignatelli, E., Corsini, E. M., Vega Beltrán, J. C., et al. 2001, MNRAS, 323, 188

Rettig, T. W., Walsh, K., \& Consolmagno, G. 2001, Icar, 154,313

Ricker, G. R., Winn, J. N., Vanderspek, R., et al. 2015, JATIS, 1, 4003

Romanishin, W., Tegler, S. C., Rettig, T. W., Consolmagno, G., \& Botthof, B. 2001, PNAS, 98, 863

Romanishin, W., Tegler, S. C., \& Consolmagno, G. J. 2010, AJ, 140, 29

Stanek, K. Z., Garnavich, P. M., Jha, S., et al. 2001, ApJ, 563,592

Straižys, V., Boyle, R. P., Janusz, R., Laugalys, V., \& Kazlauskas, A. 2013, A\&A, 554, A3

Straižys, V., Milašius, K., Boyle, R. P., et al. 2014a, AJ, 148,89

Straižys, V., Maskoliūnas, M., Boyle, R. P., et al. 2014b, MNRAS, 437, 1628

Straižys, V., Maskoliūnas, M., Boyle, R. P., et al. 2014c, MNRAS, 438, 1848

Straižys, V., Vrba, F. J., Boyle, R. P., et al. 2015, AJ, 149,161

Strassmeier, K. G., Ilyin, I., Järvinen, A., et al. 2015, AN, 336, 324

Taylor, V. A., Jansen, R. A., \& Windhorst, R. A. 2004, PASP, 116, 762

Taylor, V. A., Jansen, R. A., Windhorst, R. A., Odewahn, S. C., \& Hibbard, J. E. 2005, ApJ, 630, 784

Tegler, S. C., Romanishin, W., Consolmagno, G. J., et al. 2005, Icar, 175, 390

Tomaney, A. B. \& Crotts, A. P. S. 1996, AJ, 112, 2872

Uglesich, R. R., Crotts, A. P. S., Baltz, E. A., et al. 2004, ApJ, 612, 877

van Zee, L., Barton, E. J., \& Skillman, E. D. 2004, AJ, 128,2797

Vega Beltrán, J. C., Pizzella, A., Corsini, E. M., et al. 2001, A\&A, 374, 394

Verbiscer, A. J., Peterson, D. E., Skrutskie, M. F., et al. 2006, Icar, 182, 211

West, S. C., Nagel, R. H., Harvey, D. A., et al. 1997, SPIE, 2871, 74 